12

\title{
Обзор и классификация пространственно-временны́х векторов дискретных состояний семифазного преобразователя
}

\author{
(C) В.М. Терешкин, Д.А. Гришин, С.П. Баландин, В.В. Терешкин \\ Уфимский государственный авиационный технический университет, \\ 450008 Уфра, Россия \\ e-mail: tvm53@mail.ru
}

Поступило в Редакцию 29 апреля 2021 г.

В окончательной редакции 27 июля 2021 г.

Принято к публикации 30 июля 2021 г.

Предметом исследования являются алгоритмы управления семифазным преобразователем, реализующие пространственно-векторную модуляцию напряжения семифазного двигателя как альтернативы трехфазному двигателю в современной электротяге. При исследовании использовались элементы теории множеств, комбинаторика, метод разложения в ряд Фурье и векторный анализ. Проверка результатов исследований была реализована на специальном стенде для экспериментальных исследований пространственной векторной модуляции напряжения семифазного двигателя.

Ключевые слова: мощность математического множества логических состояний семифазного преобразователя, векторное пространство обобщенных векторов дискретных состояний, комбинации обобщенных векторов дискретных состояний напряжения семифазного двигателя.

DOI: 10.21883/JTF.2022.01.51863.131-21

\section{Введение}

В качестве двигателя в электротяге всегда применялись классические трехфазные двигатели с вращающимся полем (асинхронные и синхронные). Однако на современном уровне развития силовой электроники, преобразовательной техники, микроконтроллеров и информационных технологий симметричное многофазное напряжения может быть сформировано с любым числом фаз, а создание многофазного двигателя возможно на основе магнитной системы трехфазной машины с вращающимся полем при незначительной модернизации рабочей обмотки, поэтому электротяга на основе многофазного двигателя (число фаз более трех) технически реализуема и может найти применение, если будут неоспоримы ее преимущества перед электроприводом на основе трехфазного двигателя с вращающимся полем.

Значительный интерес к разработкам в области электротяги проявляют производители современного транспорта [1]. В настоящее время разрабатываются гибридные силовые установки с использованием электротяги для авиации, тяговые электродвигатели успешно конкурируют с тепловыми двигателями на самолетах малой авиации, в автомобильном транспорте все чаще находят применение электромобили. Известно значительное количество работ, посвященных различным аспектам векторного управления трехфазными двигателями с вращающимся полем [2-4]. Также существуют разработки электроприводов на основе, например, четырехфазного асинхронного двигателя с векторным управлением [5].

Существуют исследования, из которых следует, что при увеличении числа фаз двигателя с вращающимся полем снижается номинальный фазный ток, уменьшает- ся уровень вибраций электромагнитного происхождения, а также возможно повышение уровня надежности электропривода [6-8]. Фактором, положительно влияющим на рост уровня надежности, является количество фаз двигателя. Таким образом, одно из направлений развития современной электротяги - это создание электропривода на основе многофазного двигателя.

Значительную долю в общем объеме работ при создании электропривода на основе многофазного двигателя составляет область управления (алгоритмы, реализующие пространственно-векторную модуляцию, регуляторы векторного управления и т.д.).

При формировании симметричного многофазного напряжения преобразователем и подключении к нему многофазного двигателя имеет место режим пространственно-векторной модуляции (обще принятое сокращение - режим SVPWM). Настоящая работа посвящена исследованию алгоритмов работы семифазного преобразователя, реализующего пространственнуювекторную модуляцию семифазного двигателя.

\section{1. Постановка проблемы}

На рис. 1 изображена функциональная схема семифазного мостового преобразователя, подключенного к симметричной семифазной обмотке (ABCDEFG) двигателя с вращающимся полем, соединенной по схеме „звезда“. Преобразователь подключен к источнику питания с напряжением $U$. Анодная группа ключей обозначена нечетными числами, катодная группа ключей — четными числами. Входное напряжение преобразователя предполагается равным единице, $U=1$. 


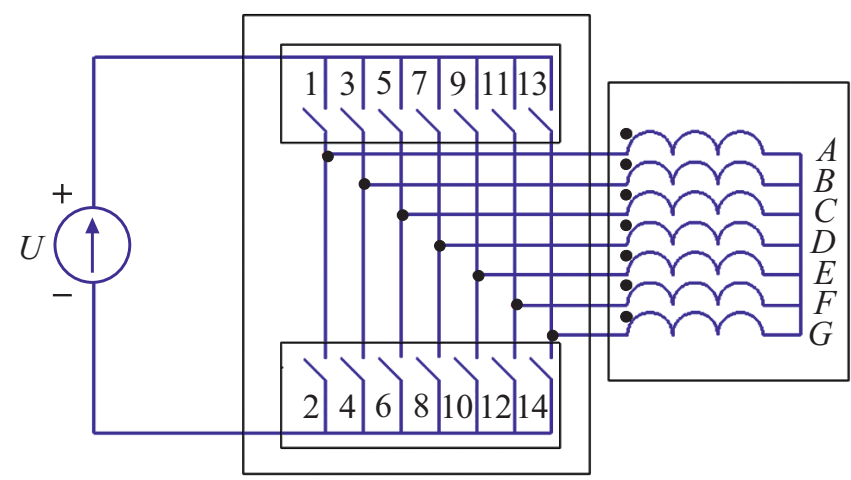

Рис. 1. Функциональная схема семифазной системы.
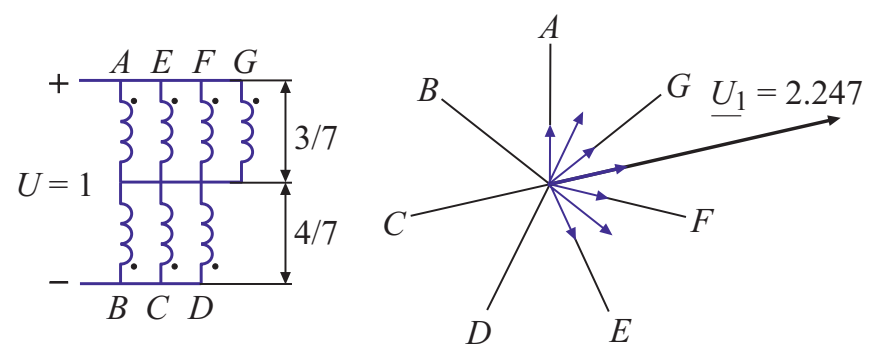

Рис. 2. Эквивалентная схема и векторное пространство напряжений семифазной обмотки при логическом состоянии преобразователя 1000111.

Семифазный преобразователь имеет $2^{7}=128$ логических состояний. Если верхний ключ плеча преобразователя замкнут, а нижний ключ разомкнут, то логическое состояние плеча преобразователя - „“. Если верхний ключ плеча преобразователя разомкнут, а нижний ключ замкнут, то логическое состояние плеча - „,“. Например, логическое состояние 1000000 соответствует замкнутому ключу 1 (при разомкнутом ключе 2) и разомкнутыми ключам 3,5,7,9 (при замкнутых ключах $4,6,8,10)$.

Логические состояния 0000000 и 1111111 соответствуют замыканию фаз семифазной обмотки нижними или верхними ключами соответственно. Таким образом, семифазный преобразователь формирует $128-2=126$ активных дискретных состояний системы, когда происходит потребление энергии двигателем, и два нулевых состояния.

Используя математическую терминологию, допустима следующая формулировка: каждое логическое состояние преобразователя является „инвариантом“, а совокупность „инвариантов“ (все возможные логические состояния преобразователя) формирует математическое множество, мощность которого равна 128.

Для сравнения мощность математического множества логических состояний трехфазного преобразователя равна 8, четырехфазного преобразователя -16 , пятифазного преобразователя - 32, шестифазного 64, а, например, 11-фазного преобразователя равна 2048.
Таким образом, при увеличении числа фаз на единицу мощность математического множества логических состояний преобразователя возрастает в 2 раза.

Каждое из 126 активных логических состояний семифазного преобразователя (каждый логический „инвариант“") формирует результирующий пространственновременной вектор напряжения семифазной обмотки (,инвариант“ векторного пространства), причем каждый вектор является вектором дискретных состояний. Процесс формирования векторов дискретных состояний напряжения принято называть пространственно-векторной модуляцией.

Каждое из двух нулевых логических состояний преобразователя может сформировать множество результирующих пространственно-временных векторов в зависимости от предыстории коммутации. Это множество векторов принято называть нулевыми векторами.

При формировании симметричного $m$-фазного напряжения происходит процесс модуляции (реализации) пространственно-временных векторов дискретных состояний. Для формирования симметричного $m$-фазного напряжения с четным числом фаз необходима $m$ ступенчатая коммутация, а для формирования симметричного $m$-фазного напряжения с нечетным числом фаз необходима $2 m$-ступенчатая коммутация.

Семифазное симметричное напряжение формируется посредством 14-ступенчатой коммутации. Множество из 126 логических состояний может быть разбито на 9 подмножеств по $14(126 / 14=9)$. Каждое из 9 подмножеств логических состояний формирует векторное пространство результирующих пространственно-временных векторов напряжения, состоящее из 14 векторов дискретных состояний. Каждое векторное пространство соответствует симметричному семифазному напряжению с определенной формой фазного напряжения.

На рис. 2 для примера приведена эквивалентная схема нагрузки преобразователя и процесс формирования результирующего вектора (дискретных состояний) в пространстве семифазной обмотки при логическом состоянии преобразователя 1000111. На рисунке изображено построение результирующего пространственновременно́го вектора напряжения дискретных состояний.

\section{2. Результаты экспериментальных исследований}

На рис. 3 приведены результаты экспериментов (верхняя часть осциллограммы - это форма фазного напряжения, нижняя часть - пульсация потенциала общей точки обмотки относительно „нуля“ преобразователя) и соответствующие векторные пространства обобщенных пространственно-временны́х векторов дискретных состояний для семифазной обмотки. В работе [9], например, приведены результаты исследований вариантов 1 и 2. 

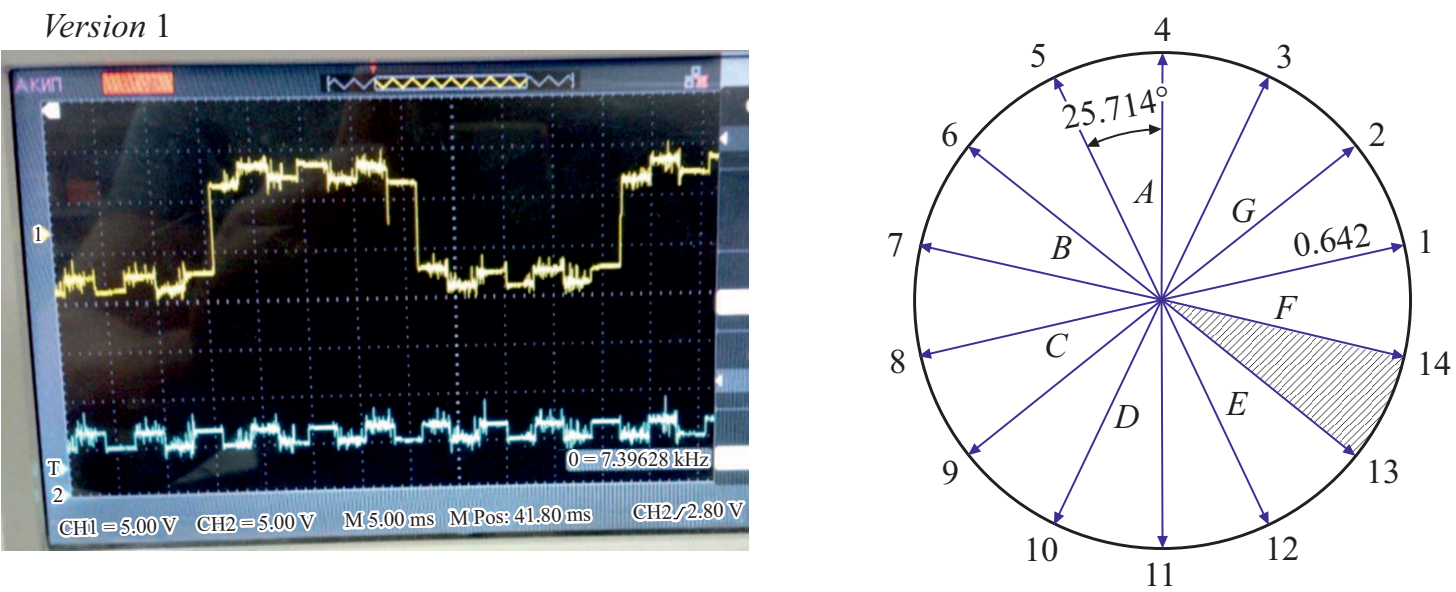

$a$

Version 2
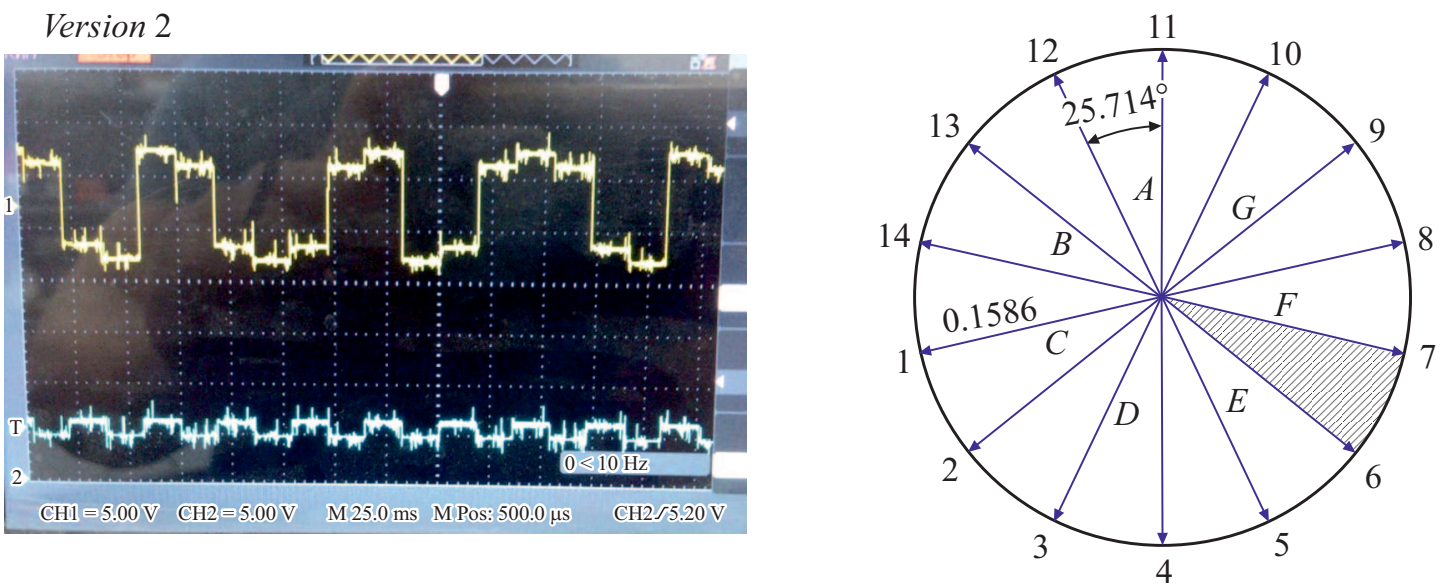

b

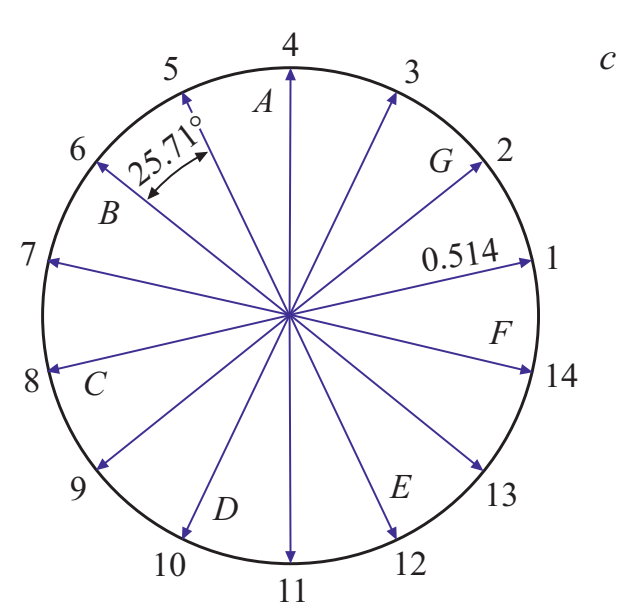

Version 3

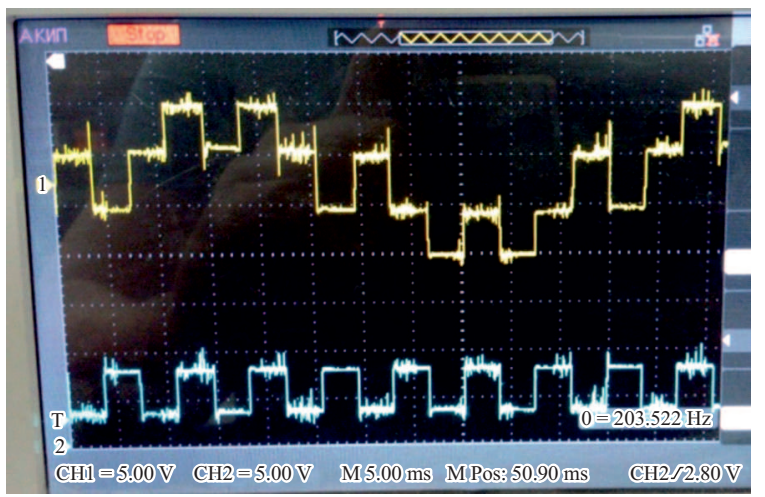

Рис. 3. Формы фазного напряжения, пульсации потенциала общей точки обмотки относительно „нуля“ преобразователя и соответствующие векторные пространства обобщенных векторов дискретных состояний. 

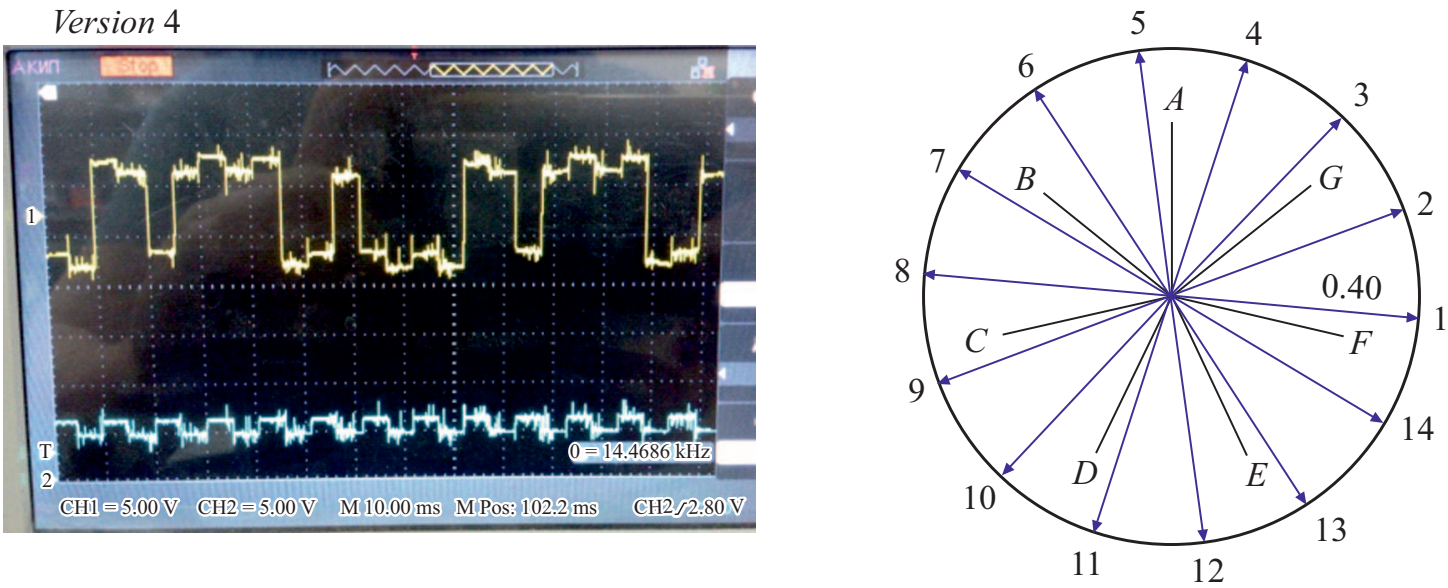

Version 5
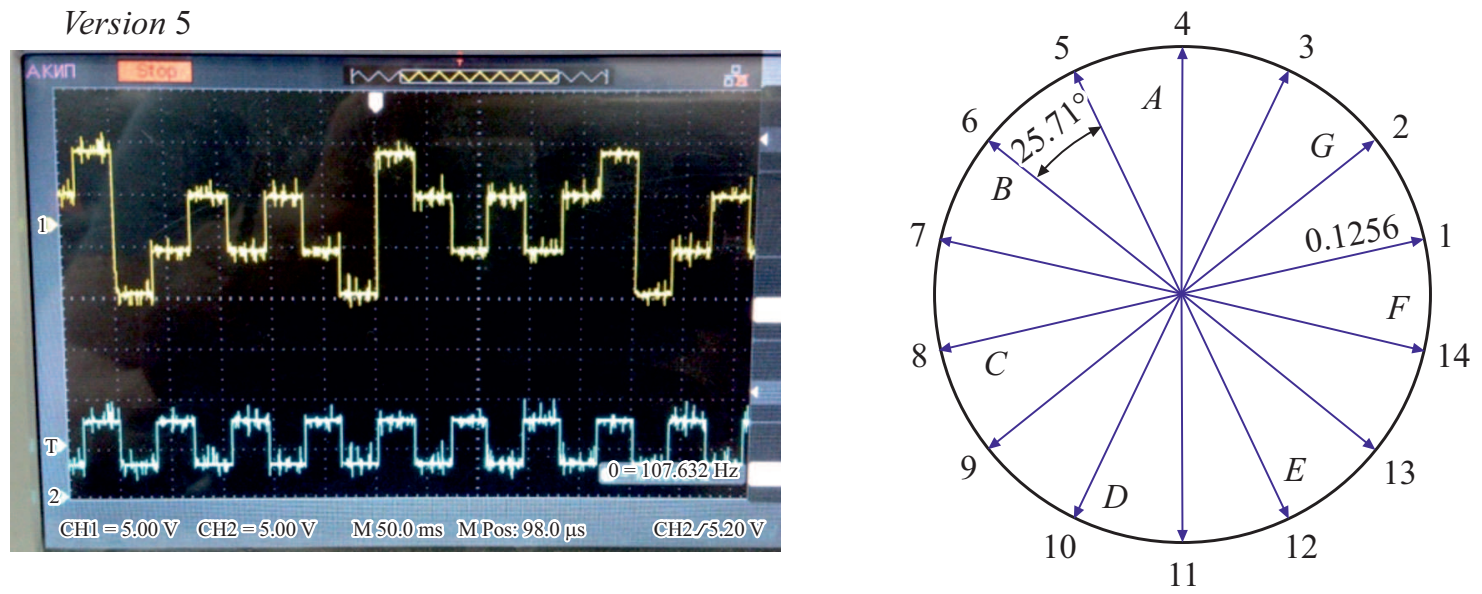

Version 6
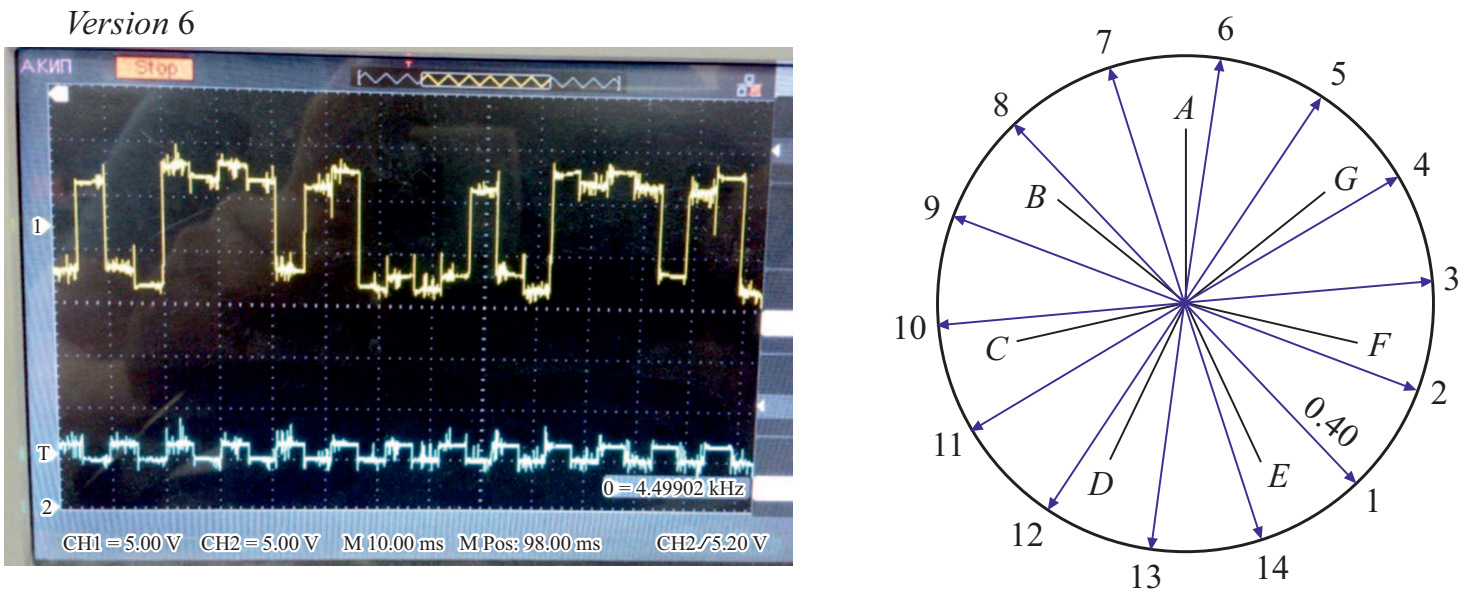

Рис. 3 (продолжение). 
Version 7

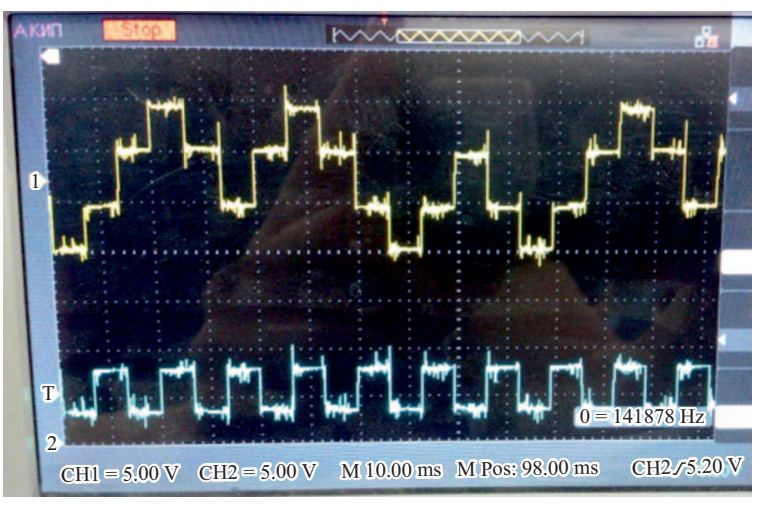

Version 8

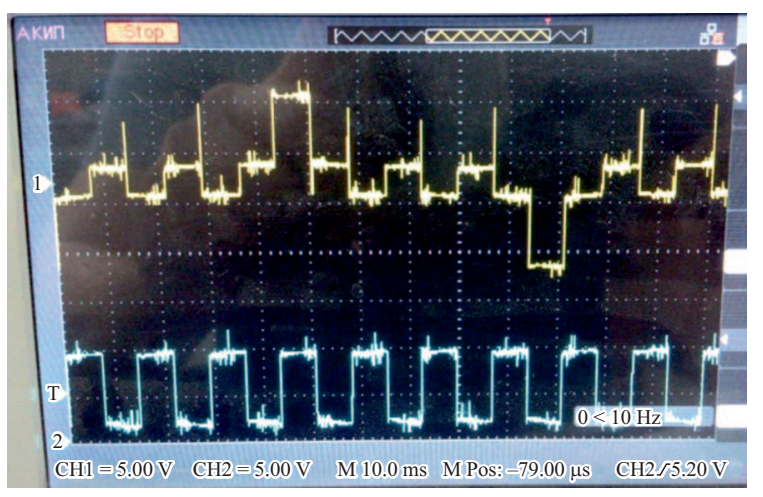

Version 9

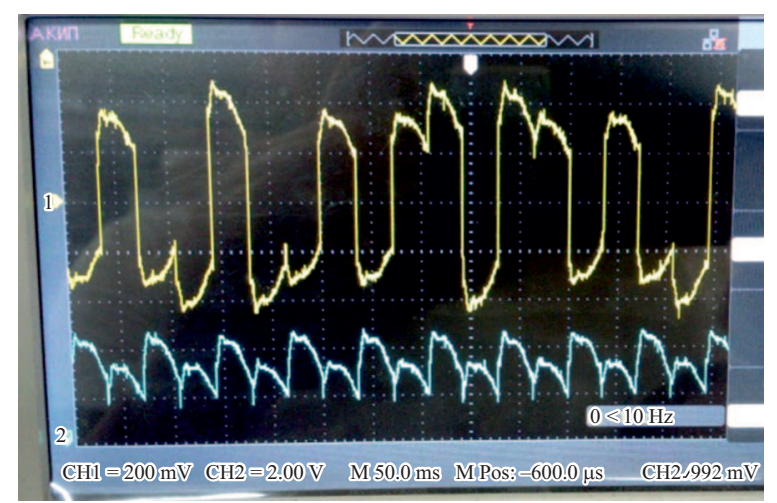

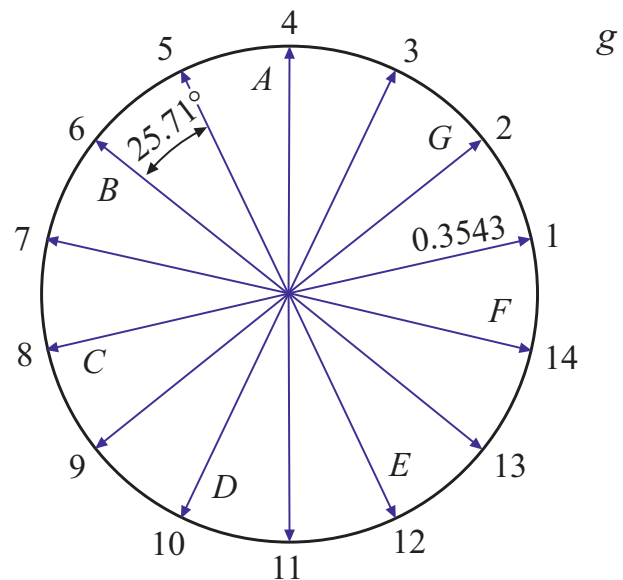
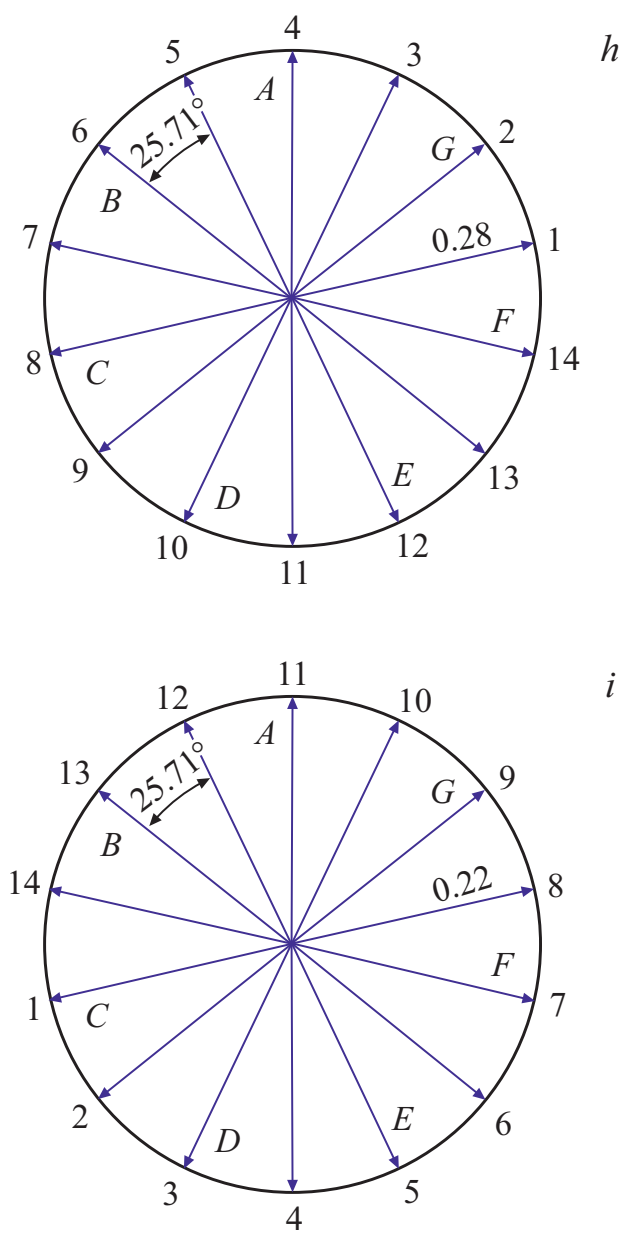

Рис. 3 (продолжение). 
На рис. 3 обозначены модули обобщенных пространственно-временны́х векторов дискретных состояний. Эти модули соответствуют амплитудам синусоид (по первой гармонике) форм фазного напряжения. Максимальный модуль вектора дискретных состояний (0.642) имеет векторное пространство варианта 1. После разложения в ряд Фурье получено, что амплитуда синусоиды по первой гармонике фазного напряжения данной формы равна 0.637. Вектор с амплитудой 0.637 является вектором непрерывного вращения.

\section{3. Логические состояния семифазного преобразователя}

Логические состояния преобразователя, которые формируют векторные пространства результирующих (обобщенных) векторов дискретных состояний, имеют вид: Вариант 1

1000111, 1000011, 1100011, 1100001, 1110001, 1110000, 1111000, 0111000, 0111100, 0011100, 0011110, 0001110, $0001111,0000111$.

14 коммутаций за период.

Вариант 2

1010100, 1011010, 0101010, 0101101, 0010101, 1010110, 1001010, 0101011, 0100101, 1010101, 1010010, 1101010, $0101001,0110101$.

$14 \cdot 3=42$ коммутации за период.

Вариант 3

0000011, 1100111, 1000001, 1110011, 1100000, 1111001, 0110000, 1111100, 0011000, 0111110, 0001100, 0011111, $0000110,1001111$.

$14 \cdot 3=42$ коммутации за период.

Вариант 4

1000110, 1001011, 0100011, 1100101, 1010001, 1110010, 1101000, 0111001, 0110100, 1011100, 0011010, 0101110, $0001101,0010111$.

$14 \cdot 3=42$ коммутации за период.

Вариант 5

1000100, 1011011, 0100010, 1101101, 0010001, 1110110, 1001000, 0111011, 0100100, 1011101, 0010010, 1101110, $0001001,0110111$.

$14 \cdot 5=70$ коммутаций за период.

Вариант 6

1001110, 0001011, 0100111, 1000101, 1010011, 1100010, 1101001, 0110001, 1110100, 1011000, 0111010, 0101100, $0011101,0010110$.

$14 \cdot 3=42$ коммутации за период.

Вариант 7

1010111, 1000010, 1101011, 0100001, 1110101, 1010000, 1111010, 0101000, 0111101, 0010100, 1011110, 0001010, $0101111,0000101$.

$14 \cdot 3=42$ коммутации за период.

Вариант 8

1101111, 0000001, 1110111, 1000000, 1111011, 0100000, 1111101, 0010000, 1111110, 0001000, 0111111, 0000100, 1011111, 0000010.
$14 \cdot 5=70$ коммутаций за период.

Вариант 9

1101100, 0011001, 0110110, 1001100, 0011011, 0100110, 1001101, 0010011, 1100110, 1001001, 0110011, 1100100, $1011001,0110010$.

$14 \cdot 5=70$ коммутаций за период.

\section{4. Исследование комбинаций векторного пространства}

Каждый из девяти вариантов имеет 6 комбинаций логических состояний преобразователя, находящихся в едином векторном пространстве. Возможные комбинации или последовательности дискретных состояний (или алгоритмы управления), которые формируют векторные пространства, имеют вид:

Комбинация $1(+1)$

$1,2,3,4,5,6,7,8,9,10,11,12,13,14 \ldots$,

$1000111,1000011,1100011,1100001,1110001,1110000$, 1111000, 0111000, 0111100, 0011100, 0011110, 0001110, $0001111,0000111$.

14 коммутаций за период.

Комбинация $2(+2)$

$1,3,5,7,9,11,13 \ldots$,

1000111, 1100011, 1110001, 1111000, 0111100, 0011110, 0001111.

$14 \cdot 2=28$ коммутаций за период.

Комбинация $3(+3)$

$1,4,7,10,13,2,5,8,11,14,3,6,9,12 \ldots$,

$1000111,1100001,1111000,0011100,0001111,1000011$, 1110001, 0111000, 0011110, 0000111, 1100011, 1110000, $0111100,0001110$.

$14 \cdot 3=42$ коммутации за период.

Комбинация $4(+4)$

$1,5,9,13,3,7,11 \ldots$,

1000111, 1110001, 0111100, 0001111, 1100011, 1111000, 0011110.

$14 \cdot 4=56$ коммутаций за период.

Комбинация $5(+5)$

$1,6,11,2,7,12,3,8,13,4,9,14,5,10 \ldots$,

1000111, 1110000, 0011110, 1000011, 1111000, 0001110, 1100011, 0111000, 0001111, 1100001, 0111100, 0000111, $1110001,0011100$.

$14 \cdot 5=70$ коммутаций за период.

Комбинация $6(6+)$

$1,7,13,5,11,3,9 \ldots$,

1000111, 1111000, 0001111, 1110001, 0011110, 1100011, 0111100.

$14 \cdot 6=84$ коммутаций за период.

В общем виде число комбинаций равно $(m-1)$, где $m-$ нечетное число фаз. Например, обмотка с числом фаз $m=11$ имеет десять комбинаций.

Экспериментальные формы фазных напряжений при реализации комбинаций приведены на рис. 4. Частота преобразователя $f$ равна $1.6 \mathrm{~Hz}$. 


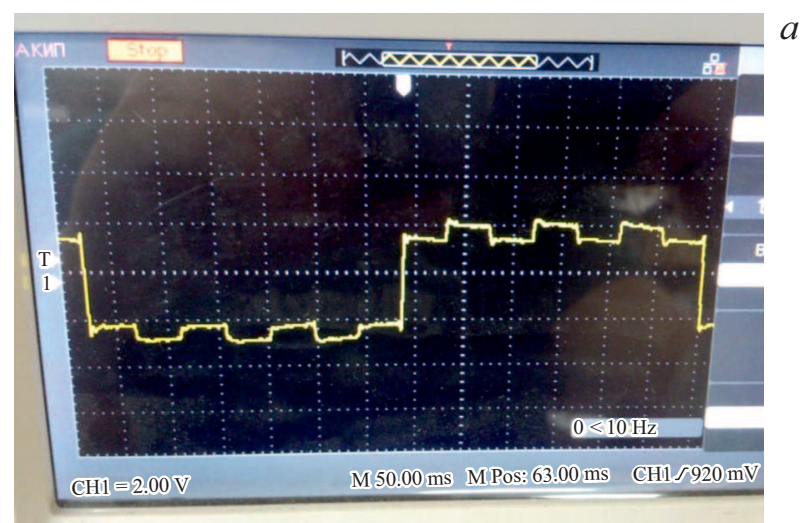

$+1$

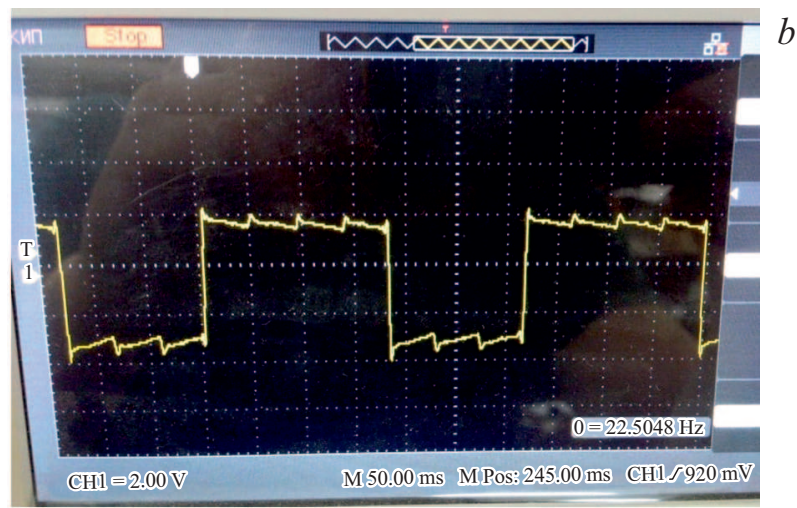

$+2$

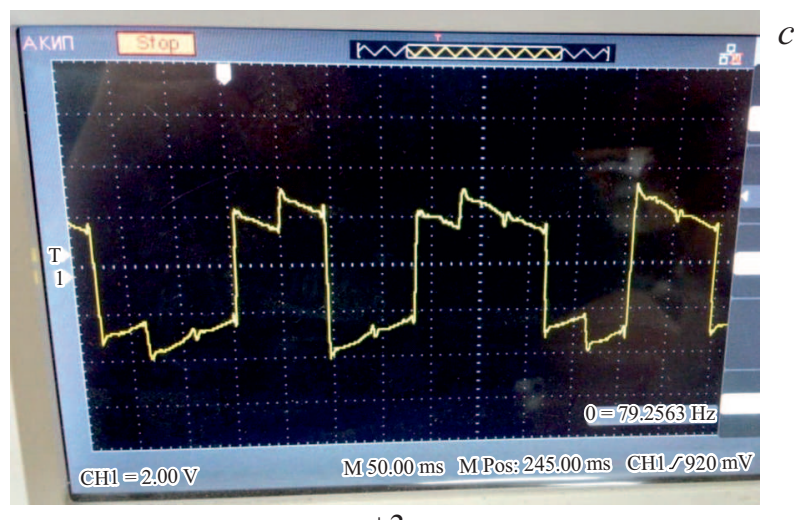

$+3$

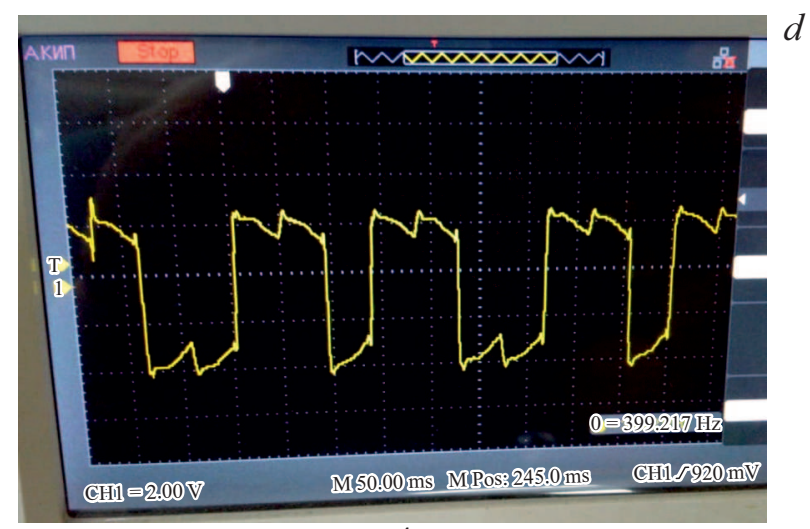

$+4$
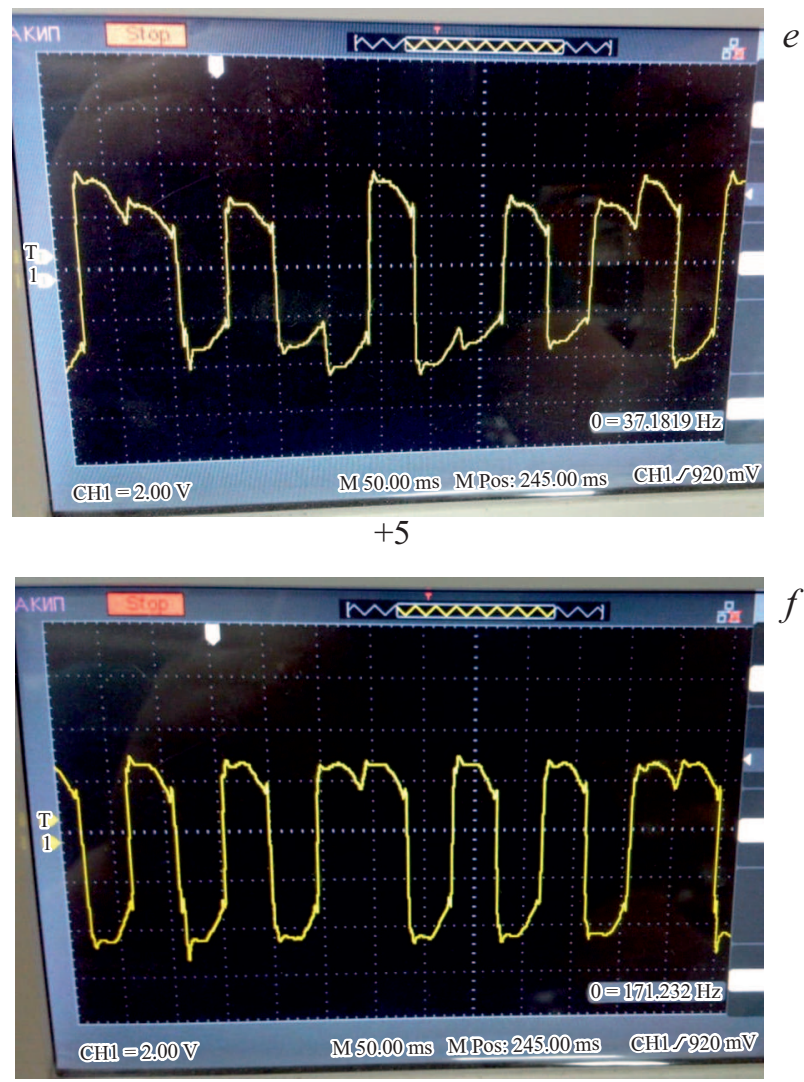

$+6$

Рис. 4. Формы фазных напряжений при реализации шести комбинаций варианта 1.

Экспериментальные результаты исследований получены с помощью стенда, созданного для исследований алгоритмов пространственно-векторной модуляции многофазных машин (число фаз от 3 до 8). Фотография стенда приведена на рис. 5. Подробное описание стенда приведено в [10].

\section{5. Обсуждение}

Мощность математического множества логических состояний семифазного преобразователя, равная 126, позволяет получить большое количество алгоритмов управления, реализующих пространственно-векторную модуляцию семифазной обмотки, формируя симметричное семифазное напряжение.

Существует девять алгоритмов, с помощью которых можно формировать симметричное семифазное напряжение с девятью формами фазного напряжения (с различным спектральным составом фазного напряжения). Возможные формы напряжения приведены на рис. 3, из которых следует, что наиболее эффективной выборкой логических состояний является выборка варианта 1. Именно выборка по варианту 1 позволяет формировать 


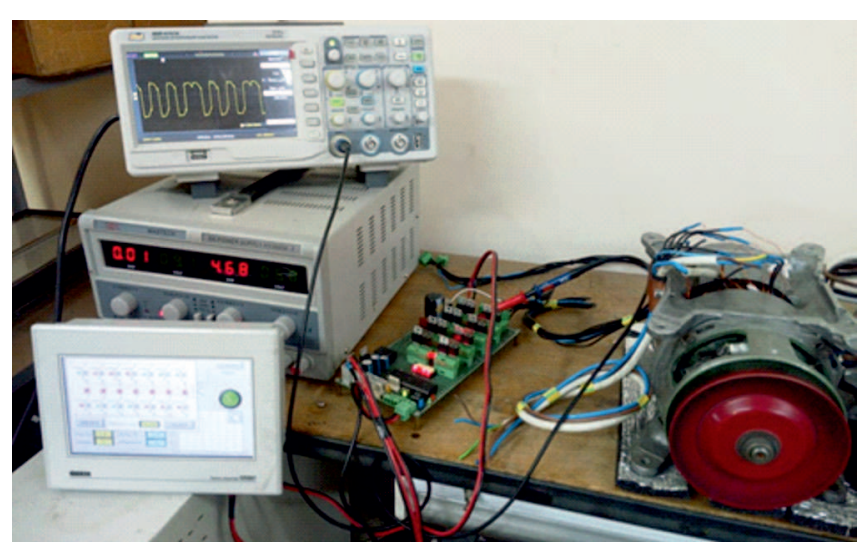

Рис. 5. Стенд для исследования алгоритмов пространственновекторной модуляции многофазных двигателей.

пространственно-временны́е векторы напряжения дискретных состояний, имеющие максимальную величину.

\section{1. Комбинация 1 выборки по варианту 1}

При реализации 14-ступенчатой коммутации в последовательности логических состояний:

$1000111,1000011,1100011,1100001,1110001,1110000$, 1111000, 0111000, 0111100, 0011100, 0011110, 0001110, 0001111, 0000111,

вектор дискретных состояний совершает один оборот за период преобразователя с шагом 25.71 electrical degree и ,формирует“ пространственно-временно́й вектор непрерывного вращения по первой гармонике с амплитудой 0.637. Частота преобразователя $f$ при реализации 14-ступенчатой коммутации соответствует частоте непрерывного вращения вектора $(\bar{w}=2 p f)$.

\section{2. Комбинация 5 выборки по варианту 1}

При реализации 14-ступенчатой коммутации в последовательности логических состояний:

1000111, 1110000, 0011110, 1000011, 1111000, 0001110, 1100011, 0111000, 0001111, 1100001, 0111100, 0000111, 1110001, 0011100,

вектор дискретных состояний совершает 5 оборотов за период преобразователя с шагом 525.71 electrical degree. Такая последовательность логических состояний „формирует“ пространственно-временно́й вектор непрерывного вращения по пятой гармонике. Частота вращения вектора в 5 раз превышает частоту преобразователя $(\bar{\omega}=5 \cdot 2 \pi f)$. При этом количество коммутаций ключей за период также возрастает в пять раз $-5 \cdot 14=70$.

Форма фазного напряжения аналогична форме по варианту 9, но временная последовательность чередования фаз при реализации данного алгоритма имеет вид ACEGBDF.

Алгоритм (последовательность логических состояний преобразователя), который реализует временную последовательность чередования фаз ACEGBDF и обеспе-

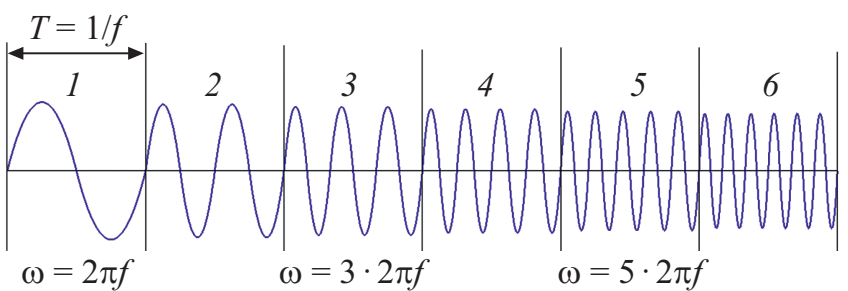

$$
\omega=2 \cdot 2 \pi f \quad \omega=4 \cdot 2 \pi f \quad \omega=6 \cdot 2 \pi f
$$

Рис. 6. Одна частота преобразователя -6 скоростей вращения вектора напряжения.

чивает форму фазного напряжения с усиленной пятой гармоникой (рис. $3, i$ ) является „алгоритмом по пятой гармонике“. Частота непрерывного вращения результирующего вектора напряжения в 5 раз больше частоты преобразователя. Модуль результирующего вектора дискретных состояний в пятой комбинации равен модулю результирующего вектора дискретных состояний в первой комбинации. Амплитуда синусоиды по пятой гармонике (рис. 3,i), или амплитуда вектора непрерывного вращения, равна 0.555 , в отличие от амплитуды синусоиды по первой гармонике (рис. 3,a), которая равна 0.637.

Аналогичные рассуждения справедливы для шести приведенных комбинаций логических состояний. Иллюстрация рассуждений приведена на рис. 6, где $T=1 / f-$ период преобразователя.

Из рис. 6 следует, что результирующий вектор непрерывного вращения семифазной симметричной обмотки имеет 6 скоростей (в общем виде $(m-1)$ скоростей при нечетном числе фаз) при одной частоте преобразователя. Так, пятифазная обмотка имеет 4 скорости, а 11фазная обмотка имеет десять скоростей. При этом двигатель работает на соответствующих гармониках фазного напряжения, которые формируются соответствующими алгоритмами (последовательностями логических состояний преобразователя).

Следует отметить, что мощность математического множества логических состояний, равная 126 , соответствует режиму коммутации преобразователя в семь ключей. Если учесть, что симметричное семифазное напряжение может быть сформировано „аккордами“, например, в шесть ключей (в этом режиме постоянно активными являются только шесть фаз двигателя), мощность множества значительно превышает 126. А стенд, изображенный на рис. 5, является эффективным инструментом для проведения экспериментальных исследований пространственно-векторной модуляции в различных режимах работы многофазных систем.

\section{Выводы}

1. С увеличением числа фаз растет мощность математического множества логических состояний преобразователя и возможное количество алгоритмов управле- 
ния, реализующих пространственно-векторную модуляцию при формировании симметричного напряжения.

2. Семифазный преобразователь при реализации режима пространственно-векторной модуляции может сформировать симметричное семифазное напряжение с девятью формами фазного напряжения. Каждая форма фазного напряжения соответствует определенному векторному пространству обобщенных векторов дискретных состояний.

3. Каждое из девяти векторных пространств имеет 6 комбинаций последовательностей чередования векторов дискретных состояний и каждая из шести комбинаций формирует результирующий вектор напряжения непрерывного вращения на определенной гармонике.

\section{Заключение}

На базе элементов теории множеств, комбинаторики, метода разложения в ряд Фурье и векторного анализа проведено исследование алгоритмов управления семифазным преобразователем, реализующих пространственно-векторную модуляцию напряжения семифазного двигателя, как альтернативы трехфазному двигателю в современной электротяге. Научная новизна исследования состоит в том, что множество активных логических состояний семифазного преобразователя (мощность математического множества равна 126) может быть разбито на 9 подмножеств. Каждое подмножество формирует симметричное семифазное напряжение с определенной формой фазного напряжения. При этом каждая форма фазного напряжения соответствует определенному векторному пространству дискретных состояний, которое состоит из 14 векторов. Последовательность чередования этих векторов имеет 6 комбинаций. Проверка результатов исследований была реализована на специальном стенде для экспериментальных исследований пространственной векторной модуляции напряжения семифазного двигателя. Результаты исследований могут быть применены при создании электротяги на основе семифазного двигателя.

\section{Конфликт интересов}

Авторы заявляют, что у них нет конфликта интересов.

\section{Список литературы}

[1] Global EV Outlook: Understanding the Electric Vehicle Landscape to 2020, Apr. 2013. [Online] Available: https:/www.ourenergypolicy.org/wp-content/uploads/2013/ 09/GlobalEVOutlook_2013.pdf

[2] В.С. Томасов, А.А. Усольцев, Д.А. Вертегел, К.М. Денисов. Научно-технический вестник информационных технологий, механики и оптики, 19 (2), 359 (2019). DOI: $10.17586 / 2226-1494-2019-19-2-359-368$.

[3] А.Е. Козярук. Электротехника, 3, 33 (2019).
[4] Y.S. Zagoskin. Adaptive Control System for Semiconductor Frequency Converters. Proceed. 2020 IEEE Conf. Rus. Young Researchers in Electrical and Electronic Engineering EIConRus. 2020. P. 960.

[5] Электровоз грузовой постоянного тока 2 ЭС10 (Гранит) с асинхронными тяговыми электродвигателями. Руководство по эксплуатации (Изд-во конструкторскоисследовательского центра ОАО „СТМ“, 2009)

[6] H. Guzman, M. Bermúdez, C. Martín, F. Barrero, M.J. Durán. Application of DSP in Power Conversion Systems A Practical Approach for Multiphase Drives, Ch. 6 in Applications of Digital Signal Processing through Practical Approach, ed. by S. Radhakrishnan (2015). DOI: $10.5772 / 59529$

[7] A. Gonzalez-Prieto, J.J. Aciego, I. GonzalezPrieto, M.J. Duran. Electronics (Switzerland), 9(6), 938 (2020). DOI: 10.3390/electronics9060938

[8] А.Н. Голубев, С.В. Игнатенко. Электротехника, 6, 28 (2000).

[9] V.M. Tereshkin, D.A. Grishin, I.I. Balgazin, V.V. Tereshkin. IEEE 2020 Intern. Confo on Electrotechnical Complexes and Systems (ICOECS), 383 (2020). DOI: 10.1109/ICOECS50468.2020.9278415

[10] В.М. Терешкин, Д.А. Гришин, И.А. Макулов. Записки Горного института, 240, 678 (2019). DOI: 10.31897/PMI.2019.6.678 